\title{
EDITORIALS
}

\section{Managing alcohol use in primary care}

Recognised problem drinking is just the tip of the iceberg.

Jim McCambridge ${ }^{1}$, Duncan Stewart ${ }^{2}$

${ }^{1}$ Addictive Behaviours and Public Health, University of York, York, UK

${ }^{2}$ Applied Health Research, London Metropolitan University, London, UK

Correspondence to: J McCambridge Jim.McCambridge@york.ac.uk

Cite this as: BMJ 2020;371:m4129 http://dx.doi.org/10.1136/bmj.m4129

Published: 25 November 2020

People who struggle with alcohol problems are stigmatised in extraordinary ways. The UK treatment system has been partly dismantled and cheaper provision outside the National Health Service accepted. ${ }^{12}$ Predictably, much of the burden of alcohol related disease is borne by hospitals, ${ }^{2}$ not least because there is little alcohol treatment available in primary care ${ }^{3}$

Even seemingly unremarkable drinking is implicated in a wide range of health issues. ${ }^{4}$ Usually unnoticed, alcohol interferes with the everyday work of primary care. ${ }^{5}$ This dysfunctional situation requires high level strategic planning ${ }^{6}$ to clarify the role of primary care in managing the UK's problematic relation with alcohol.

The linked study by Cheng and colleagues (doi:10.1136/bmj.m3934) used network meta-analysis to examine the evidence underpinning mostly drug interventions for maintaining abstinence after detoxification. ${ }^{7}$ The authors found that existing evidence for drug treatments is weak. Treatment for problem drinking usually starts before detoxification, and what to do afterwards is the focus here. The study almost entirely excluded the large literature on counselling and other psychosocial treatments $\left(\mathrm{eg}^{8}\right)$, mainly because these interventions start earlier. Some post-detoxification studies were also excluded, however, including the COMBINE study evaluating a combination of drug and behavioural interventions. ${ }^{9}$

As well as extensive risk of bias across trials, the authors found little usable information in the primary literature on the psychosocial interventions routinely offered or accessed by patients in primary care, such as Alcoholics Anonymous. This complicates interpretation of treatment effects. Abstinence is a good idea for those with severe problems, so Cheng and colleagues' focus on abstinence is appropriate. Data on other possible outcomes such as reduced consumption are not reported consistently enough for meaningful synthesis.

The authors diligently report study limitations, and their study covers its chosen ground well. Importantly, they found no reliable evidence of benefit over placebo for drugs other than acamprosate. No support is available for naltrexone and disulfiram currently recommended in UK alcohol treatment guidelines. ${ }^{10}$ Few trials in the review post-date these guidelines.

The authors recommend pragmatic trials of drugs plus interventions such as home visits to help expand primary care involvement in alcohol treatment. Prescribing is just one possible component of integrated patient centred care. ${ }^{11}$ We need to better understand which other interventions could also be offered ${ }^{12}$ and how the NHS can facilitate management of alcohol use in primary care.6 This calls for a broader perspective.

We still know little about how to help the patient stereotyped as an "alcoholic." This is despite the profound shaping of alcohol science by the alcoholism treatment movement, promoted by temperance advocates and the alcohol industry..$^{13}$ The literature on drug treatment has been biased to an unknown degree by the pharmaceutical industry. ${ }^{14}$ We should also ask why research, practice, and policy focuses largely on people with the most severe problems, rather than the important harms associated with alcohol use more broadly. 
Alcohol consumption has a predominantly dose-response relation with alcohol related harms. Alcohol use is not just a problem for drinkers. The damage done to children and families makes the problem intergenerational, ${ }^{15}$ even with normative patterns of drinking. ${ }^{16}$ The harms are greatest in socioeconomically deprived areas; alcohol expenditure reinforces household poverty and invites other adverse health consequences. ${ }^{17}$

Alcohol consumption is deeply rooted in culture, in both the UK and elsewhere. We have a paradoxical, and indeed pathological, relation with alcohol - not only because alcohol damages health and society, or because we accept high levels of avoidable harm, but because we do not possess the means to think and talk about the true balance of costs and benefits. This hinders public debate, discourages involvement in a complex poorly understood problem, and makes recovery from alcohol problems more challenging for those directly affected.

We do know that policy measures to increase price and restrict availability of alcohol help the whole population, including those with severe problems. ${ }^{18}$ But, unlike their counterparts in Scotland, Wales, and Ireland, policymakers in England continue to prioritise industry interests over public health and have not proceeded with a minimum unit price for alcohol. ${ }^{19}$

If this was any other major health problem, ${ }^{20}$ we would likely think harder, act faster, and explore more than the most visible manifestations of the problem. Scientists could provide better evidence to shape the public conversation, moving it beyond stereotypical ideas of the nature of alcohol related problems. Policymakers could better address the health, social, and economic complexities of alcohol use. Primary care could focus more on prevention, rather than on intervention after the damage is done. We must find smarter ways to explore how much alcohol lurks below the surface of common presenting problems, or risk making the already formidable challenges faced by primary care ${ }^{21}$ even more intractable.

Competing interests: The BMJ has judged that there are no disqualifying financial ties to commercial companies. The authors declare the following other interests: none.

The BMJ policy on financial interests is here:

https://www.bmj.com/sites/default/files/attachments/resources/2016/03/16-current-bmj-educationcoi-form.pdf.

Provenance and peer review: Commissioned; not externally peer reviewed.

1 Roberts E, Hotopf M, Drummond C. The relationship between alcohol-related hospital admission and specialist alcohol treatment provision across local authorities in England since passage of the Health and Social Care Act 2012. Br J Psychiatry 2020; published online 1 Jul. doi: 10.1192/bjp.2020.120 pmid: 32605664

2 Roberts E, Morse R, Epstein S, Hotopf M, Leon D, Drummond C. The prevalence of wholly attributable alcohol conditions in the United Kingdom hospital system: a systematic review, metaanalysis and meta-regression. Addiction 2019;114:1726-37. doi: 10.1111/add.14642 pmid: 31269539

3 Thompson A, Ashcroft DM, Owens L, van Staa TP, Pirmohamed M. Drug therapy for alcohol dependence in primary care in the UK: A Clinical Practice Research Datalink study. PLoS One 2017;12:e0173272. doi: 10.1371/journal.pone.0173272 pmid: 28319159

4 Stewart D, McCambridge J. Alcohol complicates multimorbidity in older adults. BMJ 2019;365:14304. doi: $10.1136 / \mathrm{bmj} .14304$ pmid: 31248871

5 Sterling SA, Palzes VA, Lu Y, etal. Associations Between Medical Conditions and Alcohol Consumption Levels in an Adult Primary Care Population. JAMA Netw Open 2020;3:e204687. doi: 10.1001/jamanetworkopen.2020.4687 pmid: 32401315

6 McCambridge J, Saitz R. Rethinking brief interventions for alcohol in general practice. BMJ 
7 Chen H-Y, McGuinness LK, Elbers RG, etal. Treatment interventions to maintain abstinence from alcohol in primary care: systematic review and network meta-analysis. BMJ 2020;371:m3934.

8 UKATT Research Team. Effectiveness of treatment for alcohol problems: findings of the randomised UK alcohol treatment trial (UKATT). BMJ 2005;331:541. doi: 10.1136/bmj.331.7516.541 pmid: 16150764

9 Anton RF, O'Malley SS, Ciraulo DA, etalCOMBINE Study Research Group. Combined pharmacotherapies and behavioral interventions for alcohol dependence: the COMBINE study: a randomized controlled trial. JAMA 2006;295:2003-17. doi: 10.1001/jama.295.17.2003 pmid: 16670409

10 National Institute for Health and Care Excellence. Alcohol-use disorders: diagnosis, assessment and management of harmful drinking (high risk drinking) and alcohol dependence (CG 115). NICE, 2011.

11 Glass JE, Bobb JF, Lee AK, etal. Study protocol: a cluster-randomized trial implementing Sustained Patient-centered Alcohol-related Care (SPARC trial). Implement Sci 2018;13:108. doi: 10.1186/s13012018-0795-9 pmid: 30081930

12 Ray LA, Meredith LR, Kiluk BD, Walthers J, Carroll KM, Magill M. Combined Pharmacotherapy and Cognitive Behavioral Therapy for Adults With Alcohol or Substance Use Disorders: A Systematic Review and Meta-analysis. JAMA Netw Open 2020;3:e208279. doi:

10.1001/jamanetworkopen.2020.8279 pmid: 32558914

13 Rubin JL. Shifting perspectives on the alcoholism treatment movement 1940--1955. J Stud Alcohol 1979;40:376-86. doi: 10.15288/jsa.1979.40.376 pmid: 381788

14 Fitzgerald N, Angus K, Elders A, etal. Weak evidence on nalmefene creates dilemmas for clinicians and poses questions for regulators and researchers. Addiction 2016;111:1477-87. doi: 10.1111/add.13438 pmid: 27262594

15 Copello A, Templeton L, Orford J, etal. The relative efficacy of two levels of a primary care intervention for family members affected by the addiction problem of a close relative: a randomized trial. Addiction 2009;104:49-58. doi: 10.1111/j.1360-0443.2008.02417.x pmid: 19133888

16 Rossow I, Felix L, Keating P, McCambridge J. Parental drinking and adverse outcomes in children: A scoping review of cohort studies. Drug Alcohol Rev 2016;35:397-405. doi: 10.1111/dar.12319 pmid: 26332090

17 Nyakutsikwa B, Britton J, Langley T. The effect of tobacco and alcohol consumption on poverty in the United Kingdom. Addiction 2020. doi: 10.1111/add.15096 pmid: 32335947

18 Babor T, Caetano R, Casswell S, etal. Alcohol, No Ordinary Commodity: Research \& Public Policy. Oxford University Press, 2010doi: 10.1093/acprof:oso/9780199551149.001.0001.

19 Hawkins B, McCambridge J. Policy windows and multiple streams: an analysis of alcohol pricing policy in England. Policy Polit 2020;48:315-33doi: 10.1332/030557319X15724461566370.

20 Marten R, Amul GGH, Casswell S. Alcohol: global health's blind spot. Lancet Glob Health 2020;8:e329-30. doi: 10.1016/S2214-109X(20)30008-5 pmid: 32087164

21 Pettigrew LM, Kumpunen S, Mays N. Primary care networks: the impact of covid-19 and the challenges ahead. BMJ 2020;370:m3353. doi: 10.1136/bmj.m3353 pmid: 32883685 